\title{
Effect of local application of curcumin and ornidazole gel in chronic periodontitis patients
}

\author{
P. L. Ravishankar, Y. Pradeep Kumar, E. N. Anila, Priyankar Chakraborty, Maharshi Malakar, R. Mahalakshmi \\ Department of Periodontics, SRM Kattankulathur Dental College, Kanchipuram, Tamil Nadu, India
}

\begin{abstract}
Objective: The objective of this study is to evaluate the comparative effect of curcumin and ornidazole in treating chronic periodontitis.

Materials and Methods: Twenty individuals of both sexes aged between 27 and 53 years diagnosed with chronic periodontitis and having pocket depths $>5 \mathrm{~mm}$ bilaterally were selected for this study, in a split-mouth design. Examination of plaque index, probing pocket depth, and clinical attachment level was measured for each patient. The patients received a complete prophylaxis including scaling and root planing after which, both test gels were injected into the two experimental sites chosen, that had probing depth (PD) $>5 \mathrm{~mm}$ and were located in symmetric quadrants. Pocket PD, clinical attachment loss, and plaque index were recorded at days 0 and 30.

Results: At 1-month evaluation, curcumin group showed a significant decrease in pocket PD, plaque index, and clinical attachment loss when compared to the ornidazole group.

Conclusion: The results show a more favorable outcome with curcumin than ornidazole gel, thus curcumin can be used as an adjunct to nonsurgical periodontal therapy.
\end{abstract}

Keywords: Curcumin, local drug delivery, ornidazole

Address for correspondence: Dr. P. L. Ravishankar, Department of Periodontics, SRM Kattankulathur Dental College, Potheri, Kanchipuram,

Tamil Nadu, India.

E-mail: Anila1992@yahoo.com

\section{INTRODUCTION}

Periodontal disease is considered as an infection that involves both inflammatory and immune responses, which leads to increased pocket depth, clinical attachment loss, as well as destruction of alveolar bone and cementum. ${ }^{[1]}$ Treatment modalities to resolve periodontal inflammation can be surgical or nonsurgical periodontal therapy based on the severity of periodontal destruction. Nonsurgical therapy includes both mechanical and chemotherapeutic methods to minimize or eliminate microbial biofilm which is the prime etiological factor of gingivitis and periodontitis.

\begin{tabular}{|l|l|}
\hline \multicolumn{2}{|c|}{ Access this article online } \\
\hline Quick Response Code: & Website: \\
\hline & www.jpionline.org \\
\cline { 2 - 3 } & \\
\hline & DOI: \\
\hline
\end{tabular}

Systemic use of antibiotics can interfere with normal body systems and may cause several side effects. The local administration of antibiotics or antimicrobials appears to be an effective means of eliminating these adverse reactions. ${ }^{[2]}$

Localized antimicrobial therapy has aroused considerable interest because of the site-specific nature of periodontal infections; the higher concentration of antimicrobial agent subgingivally, lesser the side effects of systemic antibiotic

This is an open access article distributed under the terms of the Creative Commons Attribution-NonCommercial-ShareAlike 3.0 License, which allows others to remix, tweak, and build upon the work non-commercially, as long as the author is credited and the new creations are licensed under the identical terms.

For reprints contact: reprints@medknow.com

How to cite this article: Ravishankar PL, Kumar YP, Anila EN, Chakraborty P, Malakar M, Mahalakshmi R. Effect of local application of curcumin and ornidazole gel in chronic periodontitis patients. Int J Pharma Investig 2017;7:188-92. 
use. Main advantages of local drug delivery include minimum systemic involvement, better patient compliance, and minimum discomfort. Local drug delivery releases the antimicrobial agent for an extended period at a steady pharmacological level. Among the antimicrobial agents, metronidazole, ornidazole, doxycycline, minocycline, chlorhexidine, stannous fluoride, and others have been applied subgingivally in gel forms or other sustained-release local delivery systems as varnishes, chips, or fibers. ${ }^{[3]}$

In spite of the tremendous progress in the development of medical science, plants continue to be an important source of drugs in many countries worldwide. During the past two decades, the reliability and usage of herbal products has gained increasing importance, due to the side effects and complications of many chemical and synthetic medicines. Recently, there is renewed interest in the use of various herbal or Ayurvedic medicines for oral and dental health such as neem, curcuma longa linn (turmeric), Punica granatum (pomegranate), Mangifera indica (mango), and Grita Kumara (aloe vera). Plants and natural products from time immemorial are used for their pharmacological applications, namely, anti-ulcerogenic, wound healing, anti-inflammatory, antimicrobial, and antioxidants. ${ }^{[4]} \mathrm{A}$ large number of herbal products are researched extensively for oral use in the form of mouth rinses, gels, and chips. Among various plant products, neem and Aloe vera are used widely in the treatment of oral diseases. Currently, curcumin has been generating much interest in the field of periodontics as this shows a good anti-inflammatory response.

Curcuma longa is a yellow, water-insoluble pigment extracted from the root of turmeric, a commonly used spice and food-coloring agent in Southeast Asian cooking. It has also been used as a therapeutic agent in Ayurvedic medicine for thousands of years. Recently, its anti-inflammatory, antioxidant, and anticancer effects have been studied extensively. It is also reported to have antibacterial and antifungal properties. ${ }^{[5]}$ The group nitroimidazole (metronidazole, ornidazole, etc.) is specifically an anti-anaerobic group. As anaerobic bacteria are believed to be the predominant causative factor in periodontitis and metronidazole, a member of nitroimidazole class of antibiotics specifically targets anaerobic microorganisms; it is used in the treatment of chronic periodontitis.

Metronidazole is the most common broad-spectrum antibiotic and is active against most of the periodontal pathogens. Nitroimidazole compound acts by inhibiting DNA synthesis. It works on the principle that inactive form passively diffuses into cell where it is activated by chemical reduction. The nitro group gets reduced into anion radicals which causes oxidation of DNA leading to strand breakage and cell death. Hence, it has both antimicrobial and mutagenic effects. This effect is primarily seen on obligate Gram-negative anaerobes such as Porphyromonas gingivalis, Prevotella intermedia, Fusobacterium, Selenomonas sputigina, Bacteroides forsythus, and the Grampositive anaerobes such as Peptostreptococcus and Campylobacter rectus, which are implicated in periodontal disease. ${ }^{[6]}$ To the best of our knowledge, no studies have been reported to compare the efficacy of commercially available curcumin gel and ornidazole gels in periodontitis. This study was done to evaluate and compare the clinical efficacy of these agents.

\section{MATERIALS AND METHODS}

Single-blind, randomized study was performed to assess and compare the clinical healing following single intrasulcular applications of commercially available curcumin gel (C. longa extract-10 mg) (Curenext, Abbott healthcare limited, Mumbai, India) and ornidazole gel $(1.0 \%$ ornidazole gel, chlorhexidine gluconate solution $-0.25 \%$ as preservative (Ornigreat, Mankind Pharma Limited, New Delhi, India) [Figure 1] in periodontal pockets previously treated by scaling and root planing (SRP). Sample size was determined on the basis of statistician's recommendation to achieve statistical significance, and accordingly, 20 patients (14 males and 6 females) aged 27-53 years diagnosed with chronic periodontitis and having pocket depth $>5 \mathrm{~mm}$ were selected for the study from patients who visited the Department of Periodontics, SRM Kattankulathur Dental College, Potheri, Tamil Nadu. Informed consent was obtained from all the patients. The study protocol was reviewed and approved by the Board of Ethical Committee.

\section{Inclusion criteria}

- Patients with a pocket depth of 5-7 $\mathrm{mm}$ in at least three nonadjacent sites in different quadrants of the mouth

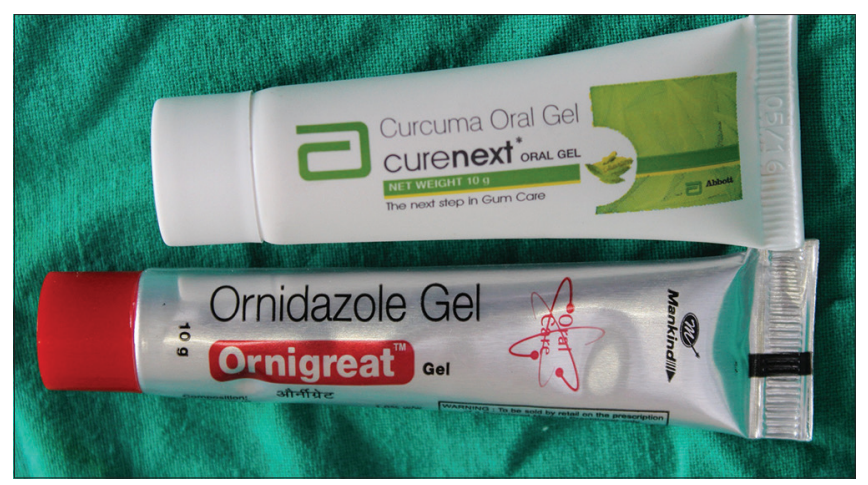

Figure 1: Commercially available curcumin and ornidazole 
- Systemically healthy controls

- Cooperative patients who could be motivated for further oral hygiene instructions

- Patients with $\geq 20$ teeth

- Patients who consented to participate in the study.

\section{Exclusion criteria}

- Patients on antibiotic therapy from the past I month

- Pregnant or lactating women

- Patients smoking tobacco.

All the participants selected in the present study received supragingival scaling and were given oral hygiene instructions before the commencement of the study. Customized acrylic stents were made for the test sites where the drug was to be placed for the standardization of the clinical parameters.

In this clinical trial, 20 patients (14 males, 6 females) were enrolled in the study. A total of 60 sites among the enrolled participants were selected for the study. Each patient had at least three teeth with probing pocket depth of $5-8 \mathrm{~mm}$ that bled on probing at the initial visit. Before starting the trial, all the patients underwent full mouth supra and subgingival scaling and root planing. ${ }^{[7]}$ After isolating with cotton rolls and drying the sites, both test gels were injected into the periodontal pocket, and periodontal dressing was given. For each participant, two experimental sites were chosen that had probing depth (PD) $>5 \mathrm{~mm}$ and were located in symmetric quadrants, and after SRP, the two sites were randomized at split-mouth level by flip of a coin and divided into two groups:

Group I: Curcumin gel was placed into the periodontal pocket until the pocket was filled [Figure 2].

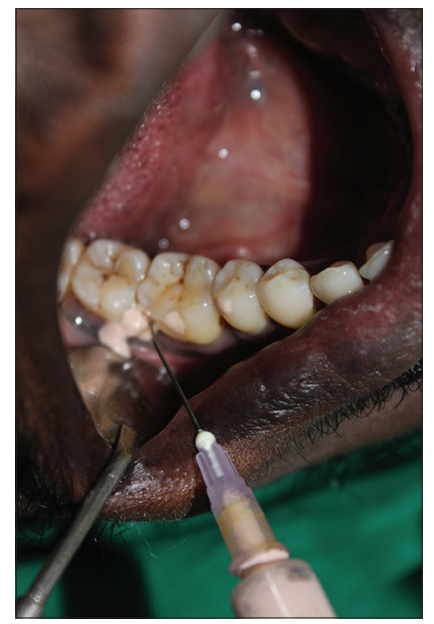

Figure 2: Subgingival delivery of curcumin
Group II: Ornidazole gel was applied directly from the syringe into the pocket, and 60 periodontal pockets were randomized into two groups before baseline. Recording of clinical parameters was carried at baseline and 1 month.

Subgingival delivery was performed with a $2 \mathrm{ml}$ disposable syringe with a blunt needle bent at its shank by $130^{\circ[8]}$ [Figure 3]. This procedure continued until the pocket was completely filled. Care was taken to apply the gel without traumatizing or damaging the periodontal tissues. After insertion of the local drug delivery system, the region was secured with a periodontal pack, and the patients were advised not to eat hard food that could traumatize the gingiva. They were also advised not to brush the treated areas for $12 \mathrm{~h}$ or floss or use interproximal cleaning devices for 10 days. They were instructed not to use any mouthwash during the study. Patients were called for the removal of periodontal dressing on $7^{\text {th }}$ day. The two groups were again examined on the $30^{\text {th }}$ day. Clinical parameters such as plaque index, probing pocket depth, and relative attachment level were recorded by single examiner who was unaware of the treatments performed on each subject. No noted adverse reactions such as pain and discomfort were observed in any participant, and healing was uneventful.

\section{Statistical analysis}

The data obtained was tabulated and analyzed statistically. The intragroup comparisons were made using paired $t$-test, and intergroup comparison was done with unpaired Student's $t$-test usingSPSS 19.0 version software.

\section{RESULTS}

All the clinical parameters were evaluated at baseline and 3 months after the nonsurgical therapy. An intragroup and intergroup comparison were made using paired $t$-test and unpaired $t$-test. Results were statistically analyzed.

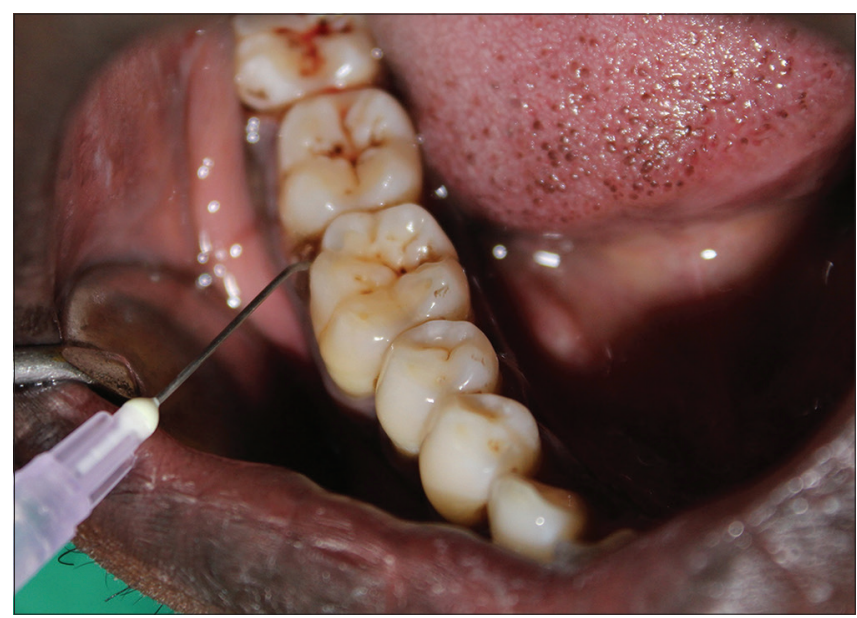

Figure 3: Subgingival delivery of ornidazole 
Table 1 shows intragroup comparison of ornidazole at baseline and 3 months using paired $t$-test. There was a significant $(P<0.001)$ decrease in PD and clinical attachment level (CAL) from $7.62+0.99$ to $5.07+1.003$ and $8.20+1.21-6.42+1.35$, respectively.

Table 2 shows intragroup comparison of curcumin at baseline and 3 months using paired $t$-test. There was a significant $(P<0.001)$ decrease in PD and CAL from $6.81+0.89$ to $3.35+0.70$ and $8.03+0.72-4.46+1.03$, respectively.

Table 3 shows intergroup comparison of curcumin and ornidazole at baseline and 3 months using unpaired $t$-test. After 3 months, curcumin group showed significantly $(P<0.001)$ better reduction in $\mathrm{PD}(3.35+0.70)$, CAL $(4.46+1.03)$, and plaque index $(0.98+0.39)$ as compared to ornidazole group (PD [5.07 + 1.003], CAL $[6.42+1.35]$, plaque index $[1.62+0.20])$.

\section{DISCUSSION}

Periodontitis is a chronic inflammatory disease caused by interplay between the subgingival microbiota and the host tissue response which leads to the destruction of supporting structures of teeth.

The standard Western medicine has had only limited success in the prevention of periodontal disease and in the treatment of a variety of oral diseases. Hence, the search for alternative products continues, and natural phytochemicals isolated from plants used in traditional medicine are considered to be good alternatives to synthetic chemicals. ${ }^{\left[{ }^{[]}\right.}$

The treatment offered to the patient by the clinician may be nonsurgical, surgical, or a combination of both. Nonsurgical therapy includes both mechanical and chemotherapeutic approaches to minimize or eliminate the microbial biofilm.

Thorough scaling and root planing (SRP) is required to prevent the recolonization of the subgingival area by periodontopathogens. However, mechanical therapy may fail to eliminate the pathogenic bacteria completely because of their location within the gingival tissues or in areas inaccessible to periodontal instrumentation.

The concept of locally delivering chemotherapeutic agents to the periodontal pocket as a method to treat periodontal disease has been studied for over few decades. Local drug delivery systems used as an adjunct to nonsurgical therapy has drastically improved the periodontal tissue condition. Day-to-day advancements in this field have led to the
Table 1: Curcumin at baseline and 1 month

\begin{tabular}{lcccc}
\hline Parameter & Baseline & 1 month & $t$ & $P$ \\
\hline PD & $6.81+0.89$ & $3.35+0.70$ & 19.8 & $<0.001$ \\
CAL & $8.03+0.72$ & $4.46+1.03$ & 6.4 & $<0.001$ \\
Plaque index & $2.53+0.27$ & $0.98+0.39$ & 7.71 & $<0.001$
\end{tabular}

Intra-group comparison. PD: Probing depth, CAL: Clinical attachment level

Table 2: Ornidazole group at baseline and 1 month

\begin{tabular}{lcccc}
\hline Parameter & Baseline & 1 month & $t$ & $P$ \\
\hline PD & $7.62+0.99$ & $5.07+1.003$ & 19.6 & $<0.001$ \\
CAL & $8.20+1.21$ & $6.42+1.35$ & 8.01 & $<0.001$ \\
Plaque index & $2.70+0.2$ & $1.62+0.20$ & 1.9 & $>0.05$ \\
\hline
\end{tabular}

PD: Probing depth, CAL: Clinical attachment level

Table 3: Comparison of curcumin and ornidazole at 1 month

\begin{tabular}{lccc}
\hline Parameter & Curcumin & Ornidazole & $P$ \\
\hline PD & $3.35+0.70$ & $5.07+1.003$ & $<0.001$ \\
CAL & $4.46+1.03$ & $6.42+1.35$ & $<0.001$ \\
Plaque index & $0.98+0.39$ & $1.62+0.20$ & 0.001
\end{tabular}

Intergroup comparison-unpaired t-test. PD: Probing depth,

CAL: Clinical attachment level

discovery of various new pharmacological agents to be used as local drug delivery systems. Several herbal drugs have now been garnering attention in the treatment of periodontal disease. ${ }^{[10]}$

The present study aimed at comparing the clinical efficacy of two local drug delivery systems in gel forms - first, group-containing ornidazole and other, group-containing curcumin in periodontitis.

Ornidazole specifically acts on Gram-negative anaerobic and facultative bacteria which are responsible for periodontal disease. When compared to metronidazole, ornidazole requires a very low minimum inhibitory concentration to inhibit the growth of periodontal pathogens. The antimicrobial activity of ornidazole has been proposed due to the reduction of nitro group to a more reactive amine that attacks microbial DNA, inhibiting further synthesis, and causing degradation of existing DNA. ${ }^{[1]]}$

It was suggested that curcumin might reduce the risk of inflammatory disorders, such as cancer and ulcer. These biological effects are attributed to its anti-inflammatory and antioxidant activities ${ }^{[12]}$ might prove beneficial in periodontal therapy, and hence, this study, comparison between curcumin and ornidazole local drug delivery as an adjunct to nonsurgical therapy was done. Intragroup comparison among curcumin and ornidazole groups at baseline and 3 months showed significant decrease in $\mathrm{PD}, \mathrm{CAL}$, and plaque index. Intergroup comparison between curcumin and ornidazole group showed a 
greater reduction in the curcumin group (decrease in PD from $6.81+0.89$ to $3.35+0.70$ ), (decrease in CAL from $8.03+0.72$ to $4.46+1.03)$, (decrease in plaque index from $2.53+0.27$ to $0.98+0.39)$ as compared to ornidazole group which had a (decrease in PD from $7.62+0.99$ to $5.07+1.003$ ), (decrease in CAL from $8.20+1.21 \mathrm{~s}$ to $6.42+1.35$ ), and (decrease in plaque index from $2.70+0.2$ to $1.62+0.20)$.

Curcuminoids (mixture of curcumin, demethoxycurcumin, and bisdemethoxycurcumin) are considered as key active constituents of $C$. longa and are reported to possess several biological activities. Numerous lines of evidence suggested that curcuminoids are potent anti-inflammatory agents working through multiple mechanisms, namely, suppression of the activation of nuclear factor-kappa $B$, inhibition of cyclooxygenase-2, downregulation of the expression of cell proliferation, antiapoptotic, and metastatic gene products. Curcuminoids have also demonstrated to modulate the proliferation and cellular response of various immune cell types, such as T-cells, B-cells, macrophages, neutrophils, natural killer cells, and dendritic cells. ${ }^{[13]}$ This may be attributed to the various properties of curcumin such as anti-inflammatory, antioxidant, antiallergic, anticarcinogenic, antimutagenic, anticoagulant, antidiabetic, antifibrotic, antiulcer, antifungal, and antibacterial. It causes shrinkage by reducing inflammatory edema and vascular engorgement of connective tissue. It promotes migration of epithelial cells to the wound area by promoting the localization of transforming growth factor- $\beta 1$ and inducing re-epithelialization. ${ }^{[8]}$ Antimicrobial properties of curcumin are likely due to its ability to inhibit bacterial lipopolysaccharide-induced cytokine expression and bacterial quorum sensing systems.

One of the main drawbacks of this study is that microbial evaluation was not carried out. However, further studies should be done toward clinical evaluation and determination of long-term efficacy of intrapocket application with curcumin and ornidazole on clinical parameters with larger sample and longer follow-up periods.

\section{CONCLUSION}

Curcumin was shown to have a more profound effect on chronic periodontitis when compared to ornidazole, thus giving us a more favorable treatment outcome when used as a local drug delivery agent. However, a study on a large scale would prove conclusive.

\section{Declaration of patient consent}

The authors certify that they have obtained all appropriate patient consent forms. In the form the patient(s) has/have given his/her/their consent for his/her/their images and other clinical information to be reported in the journal. The patients understand that their names and initials will not be published and due efforts will be made to conceal their identity, but anonymity cannot be guaranteed.

\section{Financial support and sponsorship}

Nil.

\section{Conflicts of interest}

There are no conflicts of interest.

\section{REFERENCES}

1. Sankar V. Local drug delivery for oral diseases challenges and applications. Oral Dis 2011;17:73-84.

2. Alfant $\mathrm{M}$, Walker CB. Local delivery tetracycline as a possible adjunct to conventional periodontal therapy. J Dent Res 1983;62:289.

3. Greenstein G, Polson A. The role of local drug delivery in the management of periodontal diseases: A comprehensive review. J Periodontol 1998;69:507-20.

4. Amrutesh S. Dentistry and Ayurveda - An evidence based approach. Int J Clin Dent Sci 2011;2:3-9.

5. Motterlini R, Foresti R, Bassi R, Green CJ. Curcumin, an antioxidant and anti-inflammatory agent, induces heme oxygenase- 1 and protects endothelial cells against oxidative stress. Free Radic Biol Med 2000;28:1303-12.

6. Patel B, Shah S, Kumar S. Evaluation of ornidazole gel as an adjunct to the phase I therapy. Adv Hum Biol 2014;4:21-5.

7. Singh HP, Muzammil, Sathish G, Nagendra Babu K, Vinod KS, Rao HP. Comparative study to evaluate the effectiveness of aloe vera and metronidazole in adjunct to scaling and root planing in periodontitis patients. J Int Oral Health 2016;8:374-7.

8. Varghese M, Nagarathna CV, Scaria L. Curcumin and metronidazole in periodontal therapy. Int J Res Ayurveda Pharm 2014;5:680-4.

9. Torwane NA, Hongal S, Goel P, Chandrashekar BR. Role of ayurveda in management of oral health. Pharmacogn Rev 2014;8:16-21.

10. Hosadurga RR, Rao SN, Jose J, Rompicharla NC, Shakil M, Shashidhara R, et al. Evaluation of the efficacy of $2 \%$ curcumin gel in the treatment of experimental periodontitis. Pharmacognosy Res 2014;6:326-33.

11. Greenstein $\mathrm{G}$. The role of metronidazole in the treatment of periodontal diseases. J Periodontol 1993;64:1-5.

12. Yadav SK, Sah AK, Jha RK, Sah P, Shah DK. Turmeric (curcumin) remedies gastroprotective action. Pharmacogn Rev 2013;7:42-6.

13. Chandrasekaran CV, Sundarajan K, Edwin JR, Gururaja GM, Mundkinajeddu D, Agarwal A, et al. Immune-stimulatory and anti-inflammatory activities of Curcuma longa extract and its polysaccharide fraction. Pharmacognosy Res 2013;5:71-9. 